\title{
CT Perfusion Imaging Predicts One-Month Outcome in Patients with Acute Spontaneous Hypertensive Intracerebral Hemorrhage
}

\author{
Huazhi Xu, Weijian Chen", Meihao Wang, Guoquan Cao, Yuxia Duan, Jiying Zhu \\ Department of Radiology, The Affiliated Hospital of Wenzhou Medical College, Wenzhou, China. \\ Email: "oufang11@163.com
}

Received March 18, 2013; revised April 23, 2013; accepted May 16, 2013

Copyright (C) 2013 Hua-zhi Xu et al. This is an open access article distributed under the Creative Commons Attribution License, which permits unrestricted use, distribution, and reproduction in any medium, provided the original work is properly cited.

\begin{abstract}
Purpose: Little is known about the relationship between perihematomal perfusion parameters in acute spontaneous hypertensive intracerebral hemorrhage patients and recent outcome. The purpose of this study was to evaluate the relationship between the perfusion parameters of the perihematomal brain tissue and the recent prognosis of patients with acute spontaneous hypertensive intracerebral hemorrhage (shICH) using CT perfusion (CTP) imaging. Methods: Twenty-six patients with clinical and CT diagnosed supratentorial shICH received CTP scanning within $8-19 \mathrm{~h}$ after symptom onset. At the maximum levels of the hematoma, cerebral blood flow (CBF), cerebral blood volume (CBV) and mean transit time (MTT) of perihematomal area (isodense within $1 \mathrm{~cm}$ rim of perilesion area on plain CT) and contralateral mirrored hemisphere were measured, and $\mathrm{rCBF}, \mathrm{rCBV}$, rMTT were calculated (ipsilateral/contralateral). The one-month follow-up in accordance with daily living table (Barthel index, BI) by telephone was recorded. Results: The $\mathrm{CBV}, \mathrm{CBF}$, and MTT values of perihematoma area were $(1.61 \pm 1.53) \mathrm{ml} \cdot 100 \mathrm{~g}^{-1},(16.48 \pm 12.58) \mathrm{ml} \cdot 100 \mathrm{~g}^{-1} \cdot \mathrm{min}^{-1}$, and $(9.12 \pm 2.57) \mathrm{s}$, respectively. There was significantly decreased CBV, CBF, and prolonged MTT in the perihematomal zone against contralateral hemisphere $\left(Z_{C B V}=-2.603, Z_{C B F}=-4.178, t_{M T T}=4.747\right.$, all $\left.P<0.05\right)$. Wilcoxon ranksum test displayed that perihematomal absolute values of $\mathrm{CBV}, \mathrm{CBF}$ were indicative of recent prognosis $\left(Z_{C B V}=-3.40\right.$, $Z_{C B F}=-2.98$, both $P<0.01$ ), but MTT, rCBF, rCBV, rMTT showed no correlation (all $P>0.05$ ). The areas under ROC curve (AUC) of CBV, CBF were $0.897,0.848$, respectively. When the threshold value of perihematma CBV was 1.24 $\mathrm{ml} \cdot 100 \mathrm{~g}^{-1}$, the sensitivity was $66.67 \%$ and the specificity was $100 \%$, the threshold value of CBF was $8.44 \mathrm{ml} \cdot 100$ $\mathrm{g}^{-1} \cdot \mathrm{min}^{-1}$ ), the sensitivity was $100 \%$ and the specificity was $72.70 \%$. Conclusion: CTP can predict the recent prognosis of shICH patients. Within a certain range, the recent prognosis declines with the increment of CBV CBF values.
\end{abstract}

Keywords: Cerebral Hemorrhage; X-Ray Computed Tomography; Perfusion Imaging; Outcome

\section{Introduction}

Acute spontaneously hypertensive intracerebral hemorrhage ( $\mathrm{shICH)}$ accounts for $10 \%$ to $20 \%$ of all strokes worldwide [1], and has a high disability and mortality. Variably reduced cerebral perfusion in local areas around the hematoma has been observed in reported series of patients [2-5]. The relationship between perihematoma perfusion changes and the prognosis reported less. We in-

*This study was supported by the National Key Project of Scientific and Technical Supporting Program funded by the Ministry of Science and Technology of China during the $12^{\text {th }}$ Five-Year Plan No. 2011BAI08B09. The public Welfare Science and Technology Plan Projects funded by the Science and Technology Agency of Zhejiang province of China, No. 2011C23021.

${ }^{\#}$ Corresponding author. vestigated the relation of the perihematomal hemodynamic changes and recent outcome with $\mathrm{CT}$ perfusion imaging (CTP) in shICH patients with conservative treatment.

\section{Patients and Methods}

Between January 2010 and March 2012, we prospectively examined 41 consecutive patients who were admitted due to supratentorium shICH confirmed by nonenhanced CT within 24 hours after symptom onset. Patients were excluded if they were coma; if the hemorrhage was ruptured into lateral ventricle; if there was ongoing cardiac dysfunction. Informed consent was obtained from patients or their families. All patients received multimodel CT examination (MCT), including CTP and CT 
angiography (CTA). 3 cases of arteriovenous malformation (AVM), 1 case of developmental venous malformations (DVM) comfirmed by CTA and a subgroup of 11 patients received decompressive craniotomy or hematoma evacuation were excluded from this study. The remaining 26 cases ( 22 men, 4 women; age range 33 to 74 years) received medicine treatments were included in the study. The time between the onset and MCT scan is 8 to $19 \mathrm{~h}$, the median is $15 \mathrm{~h}$.

\section{Multi-Slice Spiral CT Perfusion Imaging Protocol}

All patients were examined with a multislice CT scanner (LightSpeed 16, GE), including nonenhanced CT, CTP and CTA. Four slices perfusion images $(120 \mathrm{kV}, 200 \mathrm{~mA}$; $4 \mathrm{i} \times 5 \mathrm{~mm}$; matrix $512 \times 512)$ were acquired once every second for 50 seconds at the level of the maximum extent of the hematoma after intravenous injection of a compact bolus of $50 \mathrm{~mL}$ nonionic iodinated contrast agent (Iohexol; $300 \mathrm{mgI} / \mathrm{ml}$ ) with a power injector at a rate of 4 $\mathrm{mL} / \mathrm{s}$.

\section{Perfusion Image Analysis}

The CTP datasets were analyzed with the use of brain perfusion software 3.0 (ADW 4.2 workstation; GE). The inflow artery (anterior cerebral artery) and the outflow vein (superior sagittal sinus) were selected, cerebral blood volume (CBV) map, cerebral blood flow (CBF) map and mean transit time (MTT) map were calculated and displayed. At the maximum levels of the hematoma, $\mathrm{CBF}, \mathrm{CBV}$ and MTT of perihematomal area (isodense within $1 \mathrm{~cm}$ rim of perilesion area on nonenhanced CT) and contralateral mirrored hemisphere were measured. Then rCBF, rCBV, rMTT were calculated (ipsilateral/ contralateral). The hematoma area at the same level was manual measured using free measurement tools. The one-month follow-up of shICH patients in accordance with daily living table (Barthel index, BI) by telephone were recorded, and defined above 60 score as "good" group, less than or equal to 60 score as "poor" group.

\section{Statistical Analysis}

Data management and analysis were done with the use of the SPSS 18.0 software package. Values are given as mean $\pm \mathrm{SD}$ or median. To check for normal distribution, we used the Kolmogorov-Smirnov test and Levene test for Homogeneity of variances. Normally distributed continuous variables were analyzed with an unpaired, 2sided, Student $t$ test, and nonnormally distributed continuous variables were analyzed with the Wilcoxon ranksum test. We used multiple linear stepwise regressions for facts affecting prognosis and receiver operating characteristic curve (ROC) for threshold of perfusion parame- ters. Results were reported as significant if $\mathrm{P} \leq 0.05$.

\section{Results}

Twenty-six patients with clinically and CT diagnosed supratentorialshICH received CTP scanning within 8 $19 \mathrm{~h}$ after symptom onset were selected, including 15 cases "good" prognosis group and 11 patients "poor" group according to Barthel index score. The baseline characteristics between the two groups were summarized in Table 1. As shown in Figure 1, from the center of hematoma to normal area, the perfusion color maps showed ladder-like distribution. The $\mathrm{CBV}, \mathrm{CBF}$, and MTT value of perihematoma area was $(1.61 \pm 1.53) \mathrm{ml} \cdot 100 \mathrm{~g}^{-1}$, $(16.48 \pm 12.58) \mathrm{ml} \cdot 100 \mathrm{~g}^{-1} \cdot \mathrm{min}^{-1}$, and $(9.12 \pm 2.57) \mathrm{s}$, respectively. There was significantly decreased CBV, $\mathrm{CBF}$, and prolonged MTT in the perihematomal zone against contralateral hemisphere $\left(Z_{C B V}=-2.603, Z_{C B F}=\right.$ $-4.178, t_{M T T}=4.747$, all $\left.\mathrm{P}<0.05\right)$, see Table 2. Due to

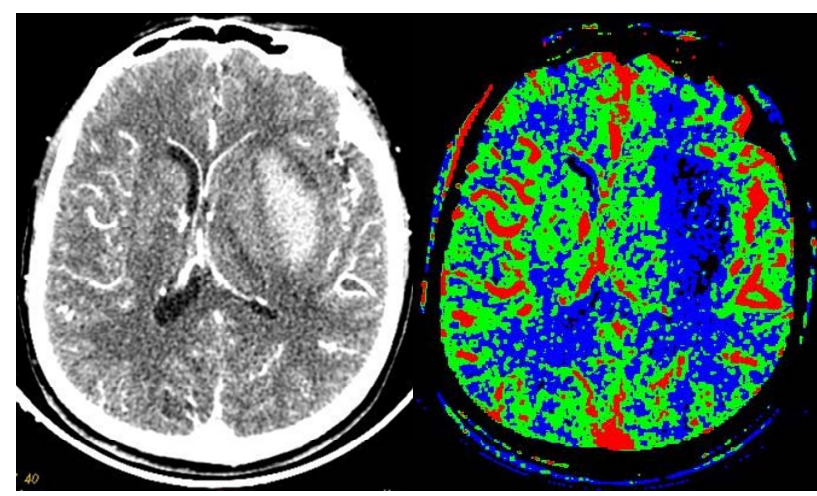

(a)

(b)

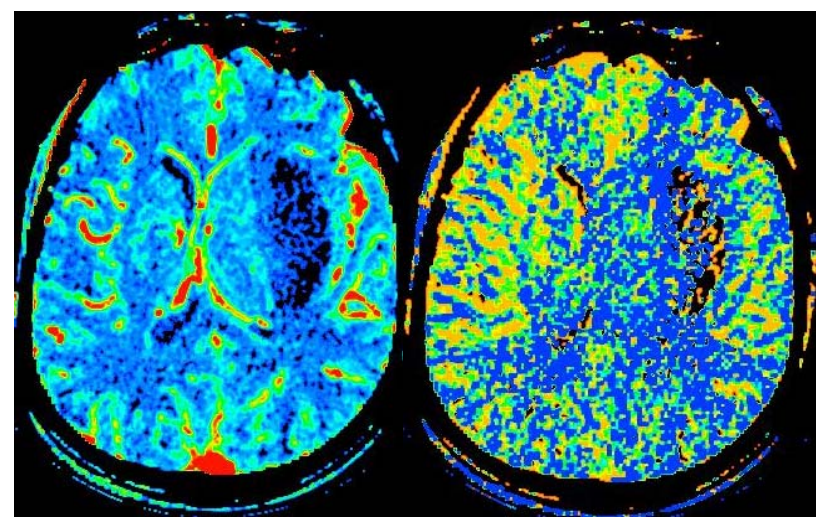

(c)

(d)

Figure 1. shICH in the left basal ganglia. A 60-year old man complained sudden weakness on the right side of limb for 13 hours. (a), Axial NECT shows massive hyperdense hematoma in the left basal ganglia, compressing the left ventricle with midline shift. (b)-(d), Color maps of $\mathrm{CBV}(\mathrm{b})$, CBF(c), MTT(d). The maps show a centrifugal distribution with a gradual increment of $\mathrm{CBV}, \mathrm{CBF}$ and prolonger MTT from the core of hematoma to the periphery. 
the small sample size, binary logical analysis was not applied for the risk factors for outcome. The difference of hematoma area between BI good group [(526.09 \pm $\left.113.97) \mathrm{cm}^{2}\right]$ and poor group $\left[(915.13 \pm 333.00) \mathrm{cm}^{2}\right]$ has statistically significant $(t=4.202, P=0.001)$. It suggested larger hematoma area predicted poor outcome.

Wilcoxon ranksum test displayed that perihematomal absolute values of $\mathrm{CBV}, \mathrm{CBF}$ were indicative of recent prognosis $\left(Z_{C B V}=-3.40, Z_{C B F}=-2.98\right.$, both $\left.P<0.01\right)$, as shown in Figure 2. But MTT, rCBF, rCBV, rMTT showed no correlation with one-month outcome (all $P>$ 0.05 ), see Table 3. The area under ROC curve (AUC) of $\mathrm{CBV}, \mathrm{CBF}$ was $0.897,0.848$, respectively. When the threshold value of perihematma $\mathrm{CBV}$ was $1.24 \mathrm{ml} \cdot 100$ $\mathrm{g}^{-1}$, the sensitivity was $66.67 \%$ and the specificity was
$100 \%$, the threshold value of CBF was $8.44 \mathrm{ml} \cdot 100$ $\left.\mathrm{g}^{-1} \cdot \mathrm{min}^{-1}\right)$, the sensitivity was $100 \%$ and the specificity was $72.70 \%$. Figure 3 and Table 4 summarized ROC parameters.

\section{Discussion}

This study described the descriptive analysis of quantitative perihematomal hypoperfusion assessed by CTP in acute shICH patients. According to our data, there was significantly decreased $\mathrm{CBV}, \mathrm{CBF}$, and prolonged MTT in the perihematomal zone against contralateral hemisphere, which was similar to most of clinical results of recent years [6-8]. Currently it was considered that hypoperfusion surrounding the hematoma was mostly due

Table 1. Patient characteristics of "good" and "poor" group.

\begin{tabular}{|c|c|c|c|c|}
\hline \multirow{2}{*}{ Patient characteristics } & “good” group & “poor” group & \multirow{2}{*}{$t / Z$} & \multirow{2}{*}{$\mathrm{P}$} \\
\hline & $\mathrm{n}=15$ & $\mathrm{n}=11$ & & \\
\hline male $(\%)$ & $11(73.3 \%)$ & $11(100 \%)$ & $Z=-1.826$ & 0.068 \\
\hline time to scan $(\mathrm{h})$ & $14.53 \pm 4.01$ & $12.18 \pm 4.14$ & $t=1.421$ & 0.168 \\
\hline hematoma area $\left(\mathrm{cm}^{2}\right)$ & $526.09 \pm 113.97$ & $915.13 \pm 333.00$ & $t=4.242$ & 0.001 \\
\hline
\end{tabular}

Table 2. CT perfusion parameters $(\bar{x} \pm s)$ of perihemotoma and contralateral mirror area and statistical test.

\begin{tabular}{ccccc}
\hline Parameter & Perihematoma & Area of mirror & Z/t value & $P$ value \\
\hline $\mathrm{CBV}\left(\mathrm{ml} \cdot 100 \mathrm{~g}^{-1}\right)$ & $1.61 \pm 1.53$ & $1.96 \pm 1.36$ & $Z=-2.603$ & 0.009 \\
$\mathrm{CBF}\left(\mathrm{ml} \cdot 100 \mathrm{~g}^{-1} \cdot \mathrm{min}^{-1}\right)$ & $16.48 \pm 12.58$ & $30.58 \pm 27.45$ & $Z=-4.178$ & $<0.001$ \\
$\mathrm{MTT}(\mathrm{s})$ & $9.12 \pm 2.57$ & $6.24 \pm 2.07$ & $t=4.747$ & 0.001 \\
\hline
\end{tabular}

Table 3. Perfusion parameters $(\bar{x} \pm s)$ of perihemotoma group by Barthel index score and statistical test.

\begin{tabular}{|c|c|c|c|c|}
\hline Parameter & “good" group & “poor” group & $Z / t$ value & $P$ value \\
\hline $\mathrm{CBV}\left(\mathrm{ml} \cdot 100 \mathrm{~g}^{-1}\right)$ & $0.83 \pm 0.31$ & $2.18 \pm 1.81$ & $Z=-3.40$ & $<0.001$ \\
\hline $\mathrm{CBF}\left(\mathrm{ml} \cdot 100 \mathrm{~g}^{-1} \cdot \mathrm{min}^{-1}\right)$ & $8.51 \pm 3.92$ & $22.33 \pm 21.00$ & $Z=-2.98$ & $<0.001$ \\
\hline MTT (s) & $9.30 \pm 2.09$ & $9.00 \pm 2.94$ & $Z=-0.60$ & $>0.05$ \\
\hline $\mathrm{rCBV}$ & $0.73 \pm 0.29$ & $0.88 \pm 0.34$ & $t=-1.06$ & $>0.05$ \\
\hline $\mathrm{rCBF}$ & $0.55 \pm 0.28$ & $0.68 \pm 0.34$ & $t=-1.38$ & $>0.05$ \\
\hline rMTT & $1.86 \pm 0.96$ & $1.55 \pm 0.90$ & $t=-1.38$ & $>0.05$ \\
\hline
\end{tabular}

Table 4. The ROC curve parameters of perihematoma CBV, CBF determined by Barthel index score.

\begin{tabular}{cccccccc}
\hline Parameter & AUC & $Z$ value & $P$ value & Cut-off value & Sensitivity & Accuracy & Specificity \\
\hline CBV & 0.897 & 6.324 & 0.001 & 1.24 & $66.67 \%$ & $66.67 \%$ & $100 \%$ \\
CBF & 0.848 & 4.579 & 0.003 & 8.44 & $100 \%$ & $72.70 \%$ & $72.70 \%$ \\
\hline
\end{tabular}



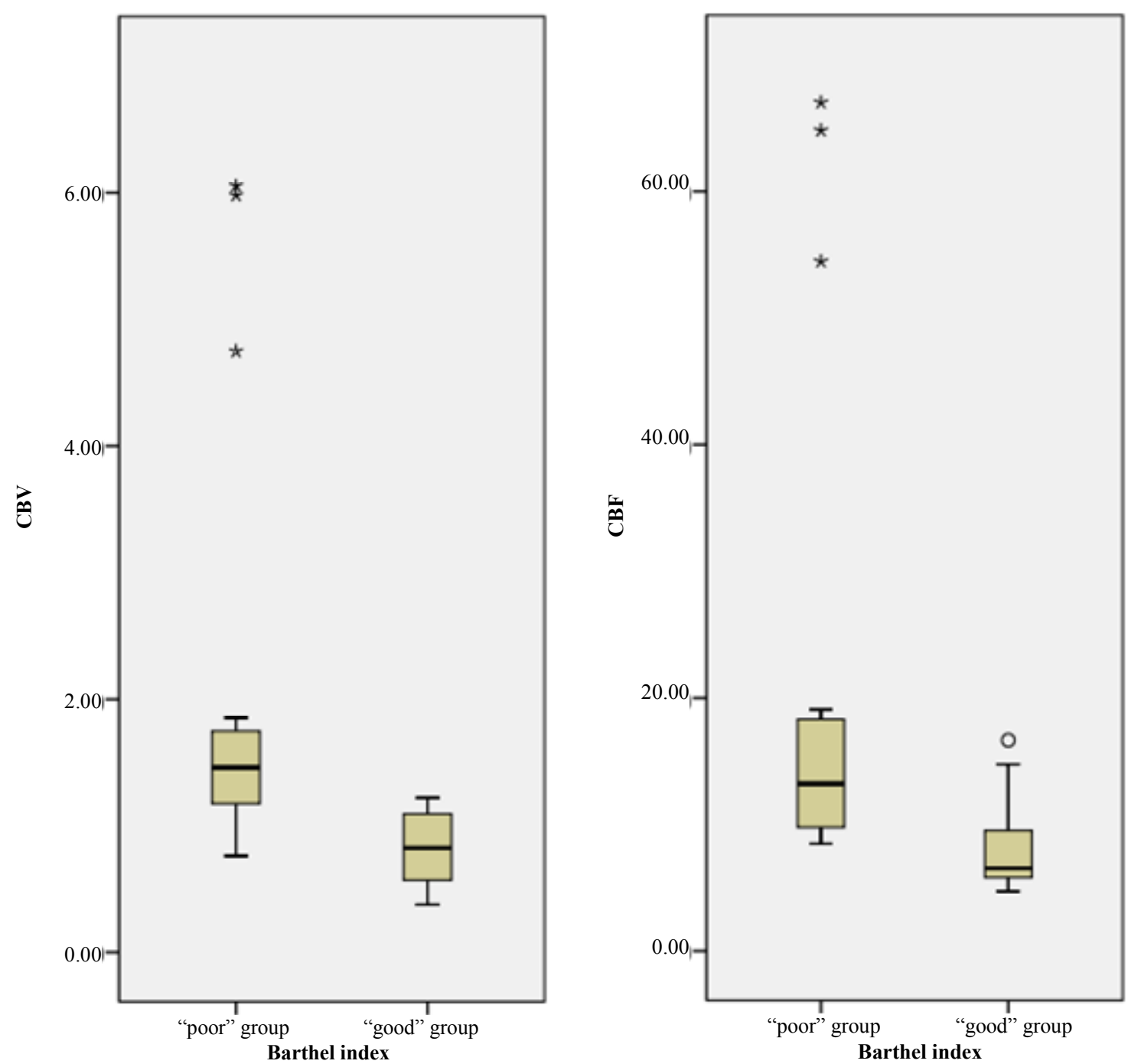

Figure 2. Boxplot of distribution of CBV, CBF value of perihematoma by BI groups. The value of CBV, CBF in "poor" group was great than that of "good" group.

to mass effect caused by the hematoma, which reduced the microcirculation volume bed and blood flow. Other reports of extravascular blood composition and its decomposition products may cause local brain tissue damage and reduce metabolic rate, which had confirmed by PET and MRI studies [9,10].

Tayal and his colleagues [11] analyzed the relationship between perihematomal CBF and in-hospital outcome in a retrospective series of 42 subacute spontaneous intracerebral hemorrhage patients undergoing xenon $\mathrm{CT}$, and consider perihematomal $\mathrm{CBF}$ independently predicts inhospital discharge status. Our data displayed the similar conclusion that perihematomal $\mathrm{CBF}$ at acute stage collated with the one-month outcome. We also reported perihematomal CBV can predict one-month outcome. What's more, the ROC curve displayed the threshold value of perihematma $\mathrm{CBV}$ was $1.24 \mathrm{ml} \cdot 100 \mathrm{~g}^{-1}$, and that of $\mathrm{CBF}$ was $8.44 \mathrm{ml} \cdot 100 \mathrm{~g}^{-1} \cdot \mathrm{min}^{-1}$. It seemed that increment of perihematomal CBV, CBF may lead the hematoma bleeding again.

Common known independent predictors of functional outcome in acute SICH are hematoma volume, Glasgow Coma Scale score, age, mass effect, and the presence of hydrocephalus [12,13]. We did not collect Glasgow Coma Scale data, and due to the small sample size, binary logical analysis was not applied for the risk factors for outcome. In our data, age was not correlated with one-month outcome, which may be associated with the small sample size or selection bias. Our study also showed larger hematoma area predict poor outcome, similar the literature reported [14]. In addition, Gebel and colleagues have previously described relative perihematomal edema volume based on CT scan as a measure that has been demonstrated to be a strong, independent predictor of 3-month functional outcome [15].

Our study had the following limitations. The sample 


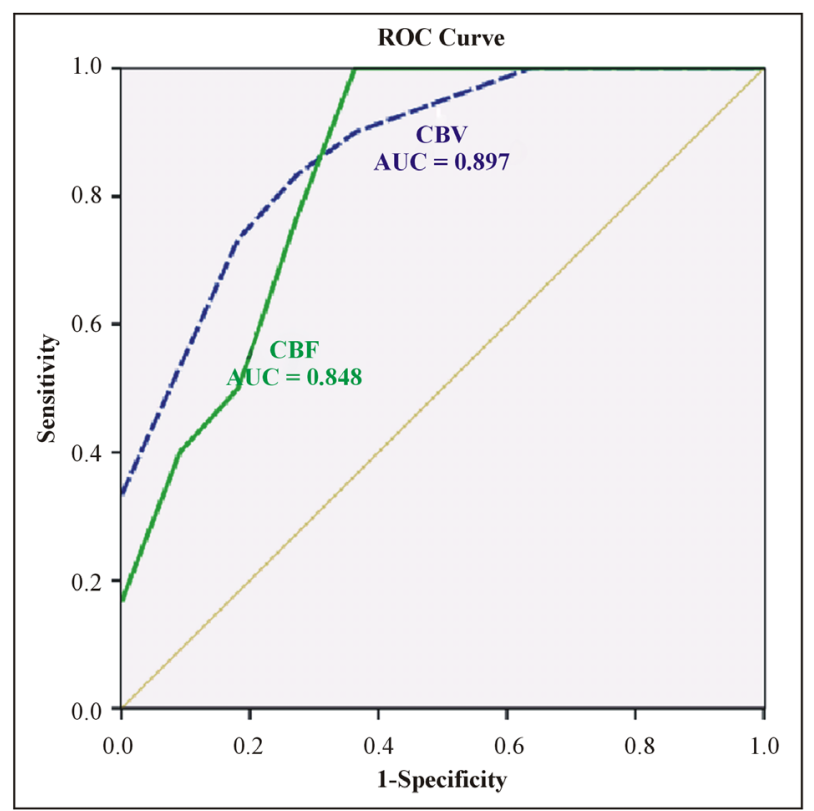

Figure 3. The ROC curve of perihematoma $\mathrm{CBV}, \mathrm{CBF}$ value by BI "good" and "poor"group.

size was small and binary logical analysis was not applied for the risk factors for outcome. Detailed, objective neurological score at admission was absent; Barthel index remains a crude marker of one-month functional outcome and was also based on multiple factors, including medical comorbidities, insurance status, and level of social support, and lack of a long time dynamic observation on shICH.

\section{REFERENCES}

[1] C. T. Skidmore and J. Andrefsky, "Spontaneous Intracerebral Hemorrhage: Epidemiology, Pathophysiology, and Medical Management," Neurosurgery Clinics of North America, Vol. 13, No. 3, 2002, pp. 281-288. doi:10.1016/S1042-3680(02)00019-0

[2] C. Herweh, E. Jüttler, P. D. Schellinger, E. Klotz, E. Jenetzky, B. Orakcioglu, et al., "Evidence against a Perihemorrhagic Penumbra Provided by Perfusion Computed Tomography," Stroke, Vol. 38, 2007, pp. 2941-2947. doi:10.1161/STROKEAHA.107.486977

[3] P. D. Schellinger, J. B. Fiebach, K. Hoffmann, K. Becker, B. Orakcioglu, R. Kollmar, et al., "Stroke MRI in Intracerebral Hemorrhage: Is There a Perihemorrhagic Penumbra?" Stroke, Vol. 34, 2003, pp. 1674-1679. doi:10.1161/01.STR.0000076010.10696.55

[4] Q. Dong, H. Y. Ding, L. F. Shi, X. Han, J. H. Fu and C. Z. $\mathrm{Lu}$, "Study of Regional Cerebral Blood Flow around Hematoma in Acute Intracerebral Hemorrhage by XenonCT," Zhonghua Yi Xue Za Zhi, Vol. 84, No. 10, 2004, pp. 822-825.

[5] R. I. Aviv, C. D. d'Esterre, B. D. Murphy, J. J. Hopyan, B. Buck, G. Mallia, V. Li, L. Zhang, S. P. Symons, T. Y.
Lee, "Hemorrhagic Transformation of Ischemic Stroke: Prediction with CT Perfusion," Radiology, Vol. 250, No. 3, 2009, pp. 867-877. doi:10.1148/radiol.2503080257

[6] M. S. Siddique, H. M. Fernandes, T. D. Wooldridge, J. D. Fenwick, P. Slomka and A. D. Mendelow, "Reversible Ischemia around intracErebral Hemorrhage: A SinglePhoton Emission Computerized Tomography Study," Journal of Neurosurgery, Vol. 96, No. 4, 2002, pp. 736-741. doi:10.3171/jns.2002.96.4.0736

[7] E. Fainardi, M. Borrelli, A. Saletti, R. Schivalocchi, C. Azzini, M. Cavallo, et al., "CT Perfusion Mapping of Hemodynamic Disturbances Associated to Acute Spontaneous Intracerebral Hemorrhage," Neuroradiology, Vol. 50, No. 8, 2008, pp. 729-740. doi:10.1007/s00234-008-0402-x

[8] N. Etminan, K. Beseoglu, B. Turowski, H. J. Steiger and D. Hänggi, "Perfusion CT in Patients with Spontaneous Lobar Intracerebral Hemorrhage: Effect of Surgery on Perihemorrhagic Perfusion," Stroke, Vol. 43, No. 3, 2012, pp. 759-763. doi:10.1161/STROKEAHA.111.616730

[9] A. R. Zazulia, M. N. Diringer, T. O. Videen, R. E. Adams, K. Yundt, V. Aiyagari, et al., "Hypoperfusion without Ischemia Surrounding Acute Intracerebral Hemorrhage," Journal of Cerebral Blood Flow \& Metabolism, Vol. 21, No. 7, 2001, pp. 804-810. doi:10.1097/00004647-200107000-00005

[10] C. S. Kidwell, J. L. Saver, J. Mattiello, S. Warach, D. S. Liebeskind, S. Starkman, et al., "Diffusion-Perfusion MR Evaluation of Perihematomal Injury in Hyperacuteintracerebral Hemorrhage," Neurology, Vol. 57, 2001, pp. 1611-1617. doi:10.1212/WNL.57.9.1611

[11] A. H. Tayal, R. Gupta, H. Yonas, T. Jovin, K. Uchino, M. Hammer, et al., "Quantitative Perihematomal Blood Flow in Spontaneous Intracerebral Hemorrhage Predicts inHospital Functional Outcome," Stroke, Vol. 38, 2007, pp. 319-324. doi:10.1161/01.STR.0000254492.35504.db

[12] M, Shaya, A, Dubey, C, Berk, E, Gonzalez-Toledo, J, Zhang, G. Caldito, et al., "Factors Influencing Outcome in Intracerebral Hematoma: A Simple, Reliable, and Accurate Method to Grade Intracerebral Hemorrhage," Surgical Neurology, Vol. 63, No. 4, 2005, pp. 343-348. doi:10.1016/j.surneu.2004.06.019

[13] B. R. Huang, C. C. Liao, W. H. Huang, Y. H. Hsu, J. C. Hsu, H. C. Yen, et al., "Prognostic Factors of Spontaneous Intracerebral Haemorrhage in Haemodialysis Patients and Predictors of 30-Day Mortality," Internal Medicine Journal, Vol. 38, No. 7, 2008, pp. 568-574. doi:10.1111/j.1445-5994.2007.01536.x

[14] K. S. Butcher, T. Baird, L. MacGregor, P. Desmond, B. Tress and S. Davis, "Perihematomal Edema in Primary Intracerebral Hemorrhage is Plasma Derived," Stroke, Vol. 35, 2004, pp. 1879-1885. doi:10.1161/01.STR.0000131807.54742.1a

[15] J. M. Gebel Jr., E. C. Jauch, T. G. Brott, J. Khoury, L. Sauerbeck, S. Salisbury, et al., "Relative Edema Volume is a Predictor of Outcome in Patients with Hyperacute Spontaneous Intracerebral Hemorrhage," Stroke, Vol. 33, 2002, pp. 2636-2641. doi:10.1161/01.STR.0000035283.34109.EA 